CERN-PPE/94-190

31 October, 1994

\title{
Experimental study of electromagnetic properties of the muon neutrino in neutrino-electron scattering
}

\author{
The CHARM II Collaboration \\ P. Vilain ${ }^{1}$, G. Wilquet ${ }^{1}$ ) \\ Inter-University Institute for High Energies (ULB-VUB), Brussels, Belgium.
}

R. Beyer ${ }^{2}$, W. Flegel, T. Mouthuy ${ }^{3}$, H. Øverås, J. Panman, K. Winter, G. Zacek ${ }^{4}$ ), V. Zacek ${ }^{5}$ )

CERN, Geneva, Switzerland.

F.W. Büsser, C. Foos, L. Gerland, T. Layda, F. Niebergall, G. Rädel ${ }^{2}$, P. Stähelin, T. Voss 2

II. Institut für Experimentalphysik ${ }^{\mathrm{a})}$, Universität Hamburg, Hamburg, Germany

D. Favart, Gh. Grégoire, E. Knoops ${ }^{6}$, V. Lemaître

Université Catholique de Louvain, Louvain-la-Neuve, Belgium

P. Gorbunov, E. Grigoriev, V. Khovansky, A. Maslennikov, A. Rozanov 3 )

Institute for Theoretical and Experimental Physics, Moscow, Russian Federation

W. Lippich, A. Nathaniel ${ }^{7}$ ), A. Staude, J. Vogt
Sektion Physik ${ }^{\text {a) }}$ der Universität München, Munich, Germany

A.G. Cocco, A. Ereditato, G. Fiorillo, F. Marchetti-Stasi, V. Palladino, P. Strolin

Università di Napoli and INFN Sezione di Napoli, Naples, Italy

A. Capone, D. De Pedis, U. Dore, A. Frenkel-Rambaldi, P.F. Loverre, D. Macina ${ }^{7}$ ), G. Piredda, R. Santacesaria

Università 'La Sapienza' and INFN Sezione di Roma, Rome, Italy

E. Di Capua, S. Ricciardi, B. Saitta

Università di Ferrara and INFN Sezione di Ferrara, Ferrara, Italy

B. Akkus, E. Arik, M. Serin-Zeyrek, R. Sever, P.Tolun

High Energy Physics Research Centre, YEFAM, Ankara, Turkey

K. Hiller, R. Nahnhauer, H. E. Roloff

DESY- Institut für Hochenergiephysik, Zeuthen, Germany

(submitted to Phys. Lett. B)

\footnotetext{
Abstract

We report new bounds on electromagnetic properties of the muon neutrino obtained from a study of neutrino-electron scattering. The results are based on the analysis of differential cross section data collected with the CHARM II detector. The $90 \%$ C.L. limit on the magnetic moment of the muon-neutrino $\mu_{\nu_{\mu}}<3 \cdot 10^{-9} \mu_{B}$ confirms limits obtained at much lower energies. The limit on the anomalous charge radius of the muon neutrino $\mid\left\langle r^{2}\right\rangle$ anom $\mid<6.0 \cdot 10^{-33} \mathrm{~cm}^{2}$ improves earlier results by nearly a factor three.

1) National Foundation for Scientific Research, Belgium

2) Now at DESY, Hamburg, Germany

3) Now at Centre de Physique des Particules, Faculté de Luminy, Marseille, France

4) Now at McGill University, Montreal, Canada

5) Now at Université de Montreal, Montreal, Canada

6) Inter-University Institute for Nuclear Science, Belgium

7) now at CERN, Geneva, Switzerland

a) supported by the German Bundesministerium für Forschung und Technologie under contract numbers 05-4HH22P and 05-4MU12P
} 


\section{Introduction}

Since the postulation of the neutrino by Pauli in 1930, its intrinsic properties have been a matter of constant interest. In recent years they became of particular interest as a possible solution of the solar neutrino problem $[1,2]$.

Although in the framework of the Standard Model, neutrinos are assumed to be electrically neutral, electromagnetic properties of the neutrino are discussed in many gauge theories beyond the Standard Model. Electromagnetic properties of the neutrino may manifest themselves as well in a magnetic moment of the neutrino as in a non vanishing charge radius, both making the neutrino subject to the electromagnetic interaction. Since the electromagnetic cross section is orders of magnitude larger than the weak cross section already very small values of an electromagnetic property could lead to observable effects. In neutrino-electron scattering they would appear as deviations from purely electroweak processes.

In this paper we report on a new search and on limits obtained from data taken with the CHARM II detector. The detector was described in detail earlier [3, 4]. It was operated during five years in the CERN SPS wide band neutrino and antineutrino beams with an average neutrino energy of about $20 \mathrm{GeV}[3,5]$. The contamination of electron-neutrinos in the muon(anti-)neutrino beam is only $1 \%$ and can safely be neglected for this analysis. More than 5000 neutrino-electron scattering events have been detected and analyzed the with main emphasis on the determination of electroweak parameters. Final results have been published recently [6].

\section{Theoretical Framework}

In the Standard Model the cross section for muon-neutrino electron scattering is given by [7]

$$
\frac{d \sigma_{\bar{\nu}}^{\nu}}{d y}=\frac{G_{F}^{2} m_{e}}{2 \pi} E_{\nu}\left[\left(g_{V} \pm g_{A}\right)^{2}+\left(g_{V} \mp g_{A}\right)^{2}(1-y)^{2}\right],
$$

where $y=E_{e} / E_{\nu}$ is the fractional energy loss of the neutrino in the laboratory system. $g_{V}$ and $g_{A}$ are the vector and axial-vector couplings of the electron to the $Z^{0}$, respectively. Kinematics is constraining the scattering angle $\theta_{e}$ of the electron by the expression $E_{e} \theta_{e}^{2}=2 m_{e}(1-y)$; this is a powerful tool to discriminate neutrino-electron scattering from the background [5].

Since the existence of a magnetic moment requires the presence of left- and right-handed neutrino states a magnetic moment of the neutrino is forbidden in the Standard Model. Evidence for a magnetic moment would therefore signal new physics. The simplest extension of the Standard Model, leads to a prediction for the magnetic moment of the neutrino of [2]

$$
\mu_{\nu}=\frac{3 G_{F} m_{e} m_{\nu}}{4 \sqrt{2} \pi^{2}} \mu_{B}=3.2 \times 10^{-19} \mu_{B} \times \frac{m_{\nu}}{\mathrm{eV}},
$$

where $\mu_{B}=e / 2 m_{e}$ is the Bohr magneton. Different models including additional physics, predict different values, as large as $10^{-10} \mu_{B}[1]$.

A neutrino-electron scattering process via a magnetic moment changes the helicity of the neutrino and this contributes a non-coherent part to the neutrino-electron scattering crosssection [8]:

$$
\frac{d \sigma^{\mu}}{d y}=\frac{\mu_{\nu}^{2}}{\mu_{B}^{2}} \frac{\pi \alpha^{2}}{m_{e} e^{2}} \frac{1-y}{y}
$$

where $y$ is defined as above. The expression is not divergent because of a lower kinematic bound of the electron energy. The integral cross-section $\sigma^{\mu}$ rises only logarithmically with the neutrino energy, while the electroweak cross section rises linearly with $E_{\nu}$. Therefore, it is advantageous to perform experiments searching for a magnetic moment of the neutrino at low neutrino energy. A low kinematic bound on the electron recoil energy improves the experimental sensitivity. In this experiment electrons with energy above $3 \mathrm{GeV}$ were selected, corresponding to an effective cut at $y \sim 0.15$. 
A non-zero charge radius of the neutrino, on the other hand, does not change the neutrino helicity and a term is therefore added coherently to the electroweak cross-section. It is expected that higher order electroweak vertex corrections do introduce a charge radius, with a magnitude depending on the top quark mass, of the order of $\left\langle r^{2}\right\rangle_{S M}=\mathcal{O}\left(10^{-33} \mathrm{~cm}^{2}\right)$ [11].

Here we define the charge radius, as it has been done in previous experiments $[9,10]$, as the derivative of the electromagnetic form factor

$$
\left\langle r^{2}\right\rangle=6 \partial f\left(q^{2}\right) /\left.\partial q^{2}\right|_{q^{2}=0}
$$

where $f$ is given by $\left\langle\nu\left|j_{\gamma}^{\mu}\right| \nu\right\rangle=f\left(q^{2}\right) \bar{u}_{\nu} \gamma^{\mu} u_{\nu}$. Both, positive and negative values of the charge radius are allowed. Here it has to be noted, that an alternative definition of the form factor $f$ [11] leads to values of the charge radius twice as large as with our definition.

An anomalous charge radius $\left\langle r^{2}\right\rangle$ anom has the effect of modifying the vector part of the interaction and effectively changes the electroweak mixing angle $\sin ^{2} \theta$ :

$$
g_{V} \rightarrow-\frac{1}{2}+2\left(\sin ^{2} \theta+\delta\right) \quad \text { with } \quad \delta=\frac{\sqrt{2} \pi \alpha}{3 G_{F}}\left\langle r^{2}\right\rangle \text { anom }
$$

Limits for the charge radius can be obtained by comparing the value of $\sin ^{2} \theta$ obtained from neutrino-electron scattering to those obtained from processes involving no neutrinos in the interaction.

Of course, effects owing to the magnetic moment and to the charge radius are not exclusive and make combined contributions to the neutrino-electron scattering amplitudes. This has to be taken into account in an experimental analysis. Throughout this paper we assumed the equality of electromagnetic properties for neutrinos and anti-neutrinos.

\section{Analysis procedure}

In this paper we followed the analysis procedure which was described in more detail before [5]. The measurement of the differential cross sections of neutrino-electron scattering in a muonneutrino and muon-anti-neutrino beam, respectively, enabled us to determine simultaneously two independent quantities. In this analysis we determined the electroweak mixing angle $\sin ^{2} \theta$ and the magnetic moment $\mu_{\nu}$ of the muon-neutrino.

The quantities $\mu_{\nu}$ and $\left\langle r^{2}\right\rangle$ anom were obtained from a fit of modelled double differential distributions in $E_{e}$ and $E_{e} \theta_{e}^{2}$ to the data. Compared to the fit presented in $[6,5]$ we added the modelled distributions for a possible magnetic moment of the neutrino according to equation (3). Using differential distributions the difference of the $y$-distributions of the electroweak and of the electromagnetic interactions can be discriminated.

For the magnetic moment of the muon-neutrino the fit yields:

$$
\mu_{\nu}=\left(1.5_{-0.6}^{+0.4} \text { (stat.) }{ }_{-1.3}^{+0.8} \text { (syst.) }\right) \times 10^{-9} \mu_{B}
$$

The correlation of the electroweak mixing angle with the magnetic moment is found to be $1.5 \%$ and can therefore be neglected.

The systematic error is dominated by the uncertainty in the neutrino flux measurement $\left({ }_{-1.1}^{+0.6}\right)$. Smaller contributions come from the uncertainty of the energy scale and of the energy resolution $\left({ }_{-0.6}^{+0.4}\right)$ and from the uncertainty of the background $( \pm 0.3)$. Adding statistical and systematical errors in quadrature the result is compatible with zero magnetic moment of the muon-neutrino, with an upper limit of

$$
\mu_{\nu}<3 \times 10^{-9} \mu_{B} \quad(90 \% \text { C.L. }) .
$$

For the electroweak mixing angle we found, in agreement with our result in [6]:

$$
\sin ^{2} \theta=0.2324 \pm 0.0062 \text { (stat.) } \pm 0.0059 \text { (syst.) }
$$


For the detailed analysis of the systematic error we refer to [6]. From this value we extract a measurement of the neutrino charge radius by comparing it to the value of the electroweak mixing angle obtained in $e^{+} e^{-}$collisions at the $Z^{0}$-resonance [12], $\sin ^{2} \Theta_{\text {eff }}^{\text {lept }}=0.2324 \pm 0.0005$. As was shown by several authors [13], the Standard Model predicts near equality of these two effective values, owing to the cancelation of higher order effects: electroweak vertex corrections contributing to the charge radius of the neutrino have the same order of magnitude but opposite sign than contributions from $(\gamma-Z)$-mixing. A difference between the two effective values can thus, at the present level of sensitivity be interpreted as an effect of an anomalous charge radius of the neutrino.

From that comparison we obtained, using eq. (5)

$$
\delta=2.38 \times 10^{30} \mathrm{~cm}^{-2}\left\langle r^{2}\right\rangle \text { anom } \leq 0.001 \pm 0.009
$$

which can be translated into a value of the anomalous charge radius of the muon-neutrino of

$$
\mid\left\langle r^{2}\right\rangle \text { anom } \mid=(0.4 \pm 3.7) \cdot 10^{-33} \mathrm{~cm}^{2}
$$

or into a limit

$$
\mid\left\langle r^{2}\right\rangle \text { anom } \mid<6.0 \cdot 10^{-33} \mathrm{~cm}^{2} \quad(90 \% \text { C.L. })
$$

\section{Conclusions}

In summary, we have determined for the first time simultaneously, bounds on the magnetic moment and the anomalous charge radius of the muon-neutrino. The limit on the anomalous charge radius establishes the best result in this domain, improving earlier results by nearly a factor of three [9], and probes substructure of the neutrino down to $|r|<7.7 \times 10^{-17} \mathrm{~cm}$ (at $90 \%$ C.L.). This result is of the same order as a charge radius arising from electroweak higher order corrections. The bound on the magnetic moment of the muon-neutrino confirms slightly better results obtained at much lower energies $[9,10]$.

\section{Acknowledgements}

We gratefully acknowledge the work of our technical collaborators who have contributed to the success of the experiment. The experiment has been made possible by grants from the Inter-University Institute for Nuclear Science (Belgium), CERN, the Bundesministerium für Forschung und Technologie (Germany), the Institute of Theoretical and Experimental Physics (Moscow, Russian Federation), and the Istituto Nazionale di Fisica Nucleare (Italy); we gratefully acknowledge their support. We thank the staff of the CERN Super Proton Synchrotron and the neutrino beam group for their competent assistance ensuring the excellent performance of their facility.

\footnotetext{
References

[1] M.B. Voloshin, M.I. Vysotskii and L.B. Okun, Zh. Eksp. Teor. Fiz. 91 (1986) 754.

[2] P. Vogel and J. Engel, Phys. Rev. D 39 (1989) 3379.

[3] CHARM II Collab., K. De Winter et al., Nucl. Inst. and Meth. A 278 (1989) 670.

[4] CHARM II Collab., D. Geiregat et al., Nucl. Inst. and Meth. A 325 (1993) 92.

[5] CHARM II Collab., D. Geiregat et al., Phys. Lett. B 281 (1992) 159.

[6] CHARM II Collab., D. Geiregat et al., Phys. Lett. B 335 (1994) 246.

[7] G. t'Hooft, Phys. Lett. B 37 (1971) 195

[8] G. Domogatskii and D. Nadezhin, Sov. J. Nucl. Phys. 12 (1971) 678.

[9] L.A. Ahrens et al., Phys. Rev. D 41 (1990) 3297; see also Particle Data Group, Phys. Rev. D 50 (1994) 1394.

[10] R.C. Allen et al., Phys. Rev. D 47 (1993) 11.

[11] G. Degrassi, A. Sirlin and W.J. Marciano, Phys. Rev. D 39 (1989) 287; J.L. Rosner, ibid. D 42 (1990) 3107.

[12] The LEP Collab.: ALEPH, DELPHI, L3 and OPAL, preprint CERN-PPE/93-157

[13] D.Yu. Bardin and V.A. Dokuchaeva, Nucl. Phys. B 246 (1984) 221; V.A. Novikov, L.B. Okun and M.I. Vysotsky, Phys. Lett. B 298 (1993) 453.
} 